\title{
Alterstice
}

Revue internationale de la recherche interculturelle

International Journal of Intercultural Research

Revista International de la Investigacion Intercultural

\section{La transmission paternelle des valeurs filiales vietnamiennes à partir du bouddhisme en France}

\section{Jérôme Gidoin}

Volume 5, numéro 1, 2015

Engagement paternel et migration

URI : https://id.erudit.org/iderudit/1077307ar

DOI : https://doi.org/10.7202/1077307ar

Aller au sommaire du numéro

Éditeur(s)

Alterstice

ISSN

1923-919X (numérique)

Découvrir la revue

Citer cet article

Gidoin, J. (2015). La transmission paternelle des valeurs filiales vietnamiennes à partir du bouddhisme en France. Alterstice, 5(1), 45-56.

https://doi.org/10.7202/1077307ar

\section{Résumé de l'article}

Cet article traite des modalités de transmission père/enfant(s), notamment père/fils aîné, dans un contexte post-migratoire, en s'appuyant sur une enquête ethnologique (2004-2008) consacrée à la relation qu'entretiennent les Vietnamiens avec leurs défunts en France dans des pagodes de la région parisienne. Au Vietnam, le père jouait un rôle social et symbolique essentiel en tant que chef de famille, et souvent " chef du culte des ancêtres ". Dans un contexte d'acculturation où le culte des ancêtres traditionnel s'effrite, on constate que l'affiliation à une association bouddhique devient une option qui permet à de nombreuses familles de faire face à une remise en question, de la part des jeunes générations, du modèle ritualiste de la transmission. On observe alors diverses formes de redéfinitions du rôle paternel par l’intermédiaire du référent bouddhique en France. 
ARTICLE THÉMATHIQUE

\section{La transmission paternelle des valeurs filiales vietnamiennes à partir du bouddhisme en France}

Jérôme Gidoin ${ }^{1}$

\section{Résumé}

Cet article traite des modalités de transmission père/enfant(s), notamment père/fils aîné, dans un contexte postmigratoire, en s'appuyant sur une enquête ethnologique (2004-2008) consacrée à la relation qu'entretiennent les Vietnamiens avec leurs défunts en France dans des pagodes de la région parisienne. Au Vietnam, le père jouait un rôle social et symbolique essentiel en tant que chef de famille, et souvent "chef du culte des ancêtres ". Dans un contexte d'acculturation où le culte des ancêtres traditionnel s'effrite, on constate que l'affiliation à une association bouddhique devient une option qui permet à de nombreuses familles de faire face à une remise en question, de la part des jeunes générations, du modèle ritualiste de la transmission. On observe alors diverses formes de redéfinitions du rôle paternel par l'intermédiaire du référent bouddhique en France.

\section{Rattachement de l'auteur}

${ }^{1}$ Centre d'anthropologie culturelle de Paris-Descartes, Paris, France

\section{Correspondance}

j.gidoin@orange.fr

\section{Mots clés}

transmission, père, filiation, bouddhisme vietnamien, hommage aux défunts, contexte post-migratoire

\section{Pour citer cet article}

Gidoin, J. (2015). La transmission paternelle des valeurs filiales vietnamiennes à partir du bouddhisme en France. Alterstice, 5(1), 45-56. 


\section{Introduction}

Une étude ethnologique (2004-2008) sur la relation aux défunts des Vietnamiens en région parisienne, réinterprétée à partir du bouddhisme, $\mathrm{m}$ 'a permis de constater que de plus en plus de familles vietnamiennes, par commodité, transfèrent leur culte des ancêtres domestique aux moines de certaines pagodes vietnamiennes (celles de Joinville-le-Pont, Bagneux, Évry). Ce phénomène résulte en quelque sorte d'une érosion du culte des ancêtres traditionnel. II faut savoir, d'une part, que ce dernier tenait une place fondamentale dans la culture vietnamienne (Condominas, 1953, p. 558-560), véhiculant les valeurs cardinales du vivre ensemble, en famille et en société et, d'autre part, que la famille vietnamienne étant de type patriarcal (Durand et Huard, 2010, p. 91), le père jouait un rôle social et symbolique essentiel, étant considéré comme le chef du culte des ancêtres au sein de la maisonnée, et au sein du groupe familial étendu s'il était le fils aîné (Cadière, 1992, p. 53-65).

Dans le contexte post-migratoire, parmi les parents qui sont nés et qui ont vécu au Vietnam, on en rencontre souvent qui ont le sentiment de ne plus parvenir à transmettre leurs valeurs culturelles. Lorsque l'on s'entretient avec les jeunes, on constate en effet à travers leurs discours une différence manifeste entre leur univers culturel et celui de leurs parents et grands-parents (Gidoin, 2014b). Se pose alors la question de la transmission de l'identité ethnico-religieuse vietnamienne d'une génération à l'autre. L'affiliation à une association bouddhique se présente alors comme une option permettant aux familles de faire face à une remise en question, de la part des jeunes générations, d'un certain modèle ritualiste de la transmission.

Les familles et l'« institution » bouddhique (les religieux et les bénévoles de l'association de la pagode) partagent une certaine idée de la continuité culturelle, en ce qui concerne notamment le lien social et le lien de filiation «à la vietnamienne ». Une continuité symbolisée par la perpétuation des hommages aux ancêtres à la pagode qui, bien qu'étant amenés à se transformer avec le temps, véhiculent les éléments essentiels d'une trame éthique en cours de redéfinition. On observe ainsi diverses formes de réinterprétation de l'identité vietnamienne à partir du référent bouddhique, sans que cela passe par une perpétuation du ritualisme traditionnel marqué par le confucianisme. Apparaissent de nouvelles manières d'appréhender les références collectives, de nouvelles modalités d'adhésion et d'identification au culturel et au religieux, et corrélativement, une redéfinition de l'affiliation à la mémoire léguée par les parents.

Dans ce contexte, il s'agit de s'interroger sur l'engagement des pères dans la transmission culturelle et religieuse. Plus spécifiquement, de quelles manières participent-ils à ce processus d' " ethnicisation " assez inédit visant à faire le lien entre le passé et le présent? II s'agira de rendre compte de la réactualisation, sous de multiples formes, du référent ethnico-religieux, et de voir quels en sont les effets au niveau de la construction subjective et identitaire des individus. Peut-on parler d'une redéfinition du rôle paternel via le référent bouddhique en France?

Je m'appuie sur des données issues d'entretiens approfondis réalisés à partir de questions relativement ouvertes posées en situation, c'est-à-dire dans le contexte d'interactions sociales observé. Les extraits restitués paraîtront peut-être abondants, mais cela m'a semblé nécessaire pour étayer mon propos et lui donner vie. Ces entretiens, de type "compréhensif " (Kaufmann, 2004), sont naturellement complétés par des entretiens informels propres à toute "enquête participante ". J'ai en particulier observé des personnes fréquentant régulièrement la pagode, d'abord de façon indirecte, puis plus systématiquement lorsque j'ai commencé à m'intéresser à la manière dont ils assimilaient et réinterprétaient la culture vietnamienne et bouddhique. Différents points de vue sur l'engagement paternel seront donnés : celui du père lui-même bien entendu - ou plutôt des pères, car selon la génération à laquelle ils appartiennent, ils entretiennent un rapport différent à la culture vietnamienne en terre d'exil -, celui de l'épouse et enfin celui des enfants, en particulier des fils aînés. Ma réflexion ne prétend nullement à l'exhaustivité, mais plus modestement à une certaine typicité, en faisant un va-et-vient entre le particulier et le général.

Je vais avoir l'occasion de développer au fil du texte un certain nombre de considérations méthodologiques et théoriques, en ce qui concerne notamment les conditions d'observation et les modalités d'entretien. Je voudrais insister au préalable sur deux points. D'abord, j'ai souvent constaté un décalage entre ce que les gens disent et ce qu'ils font, un jeu subtil entre les normes et les pratiques, qui dissimule le plus souvent des stratégies sociales liées 
à divers enjeux : identitaire, de pouvoir, etc. Aussi l'intérêt de la démarche ethnologique réside-t-il, selon moi, dans le fait de rester longtemps sur le terrain, de manière à ce que les données d'observations et d'entretiens interagissent entre elles, en se recoupant, en se complétant, voire en se contredisant. D'autre part, je revendique une démarche interprétative. Je pense en effet qu'il faut renoncer à l'idée d'objectivité au sens classique, c'est-àdire à l'idéalisation d'une connaissance prétendument objective, comme dans les sciences expérimentales, et adopter l'idée plus modeste d'une vérité plausible (Olivier de Sardan, 2013, p. 11). La socio-anthropologie est une science artisanale qui relève davantage du descriptif que de l'explicatif.

\section{Le statut traditionnel du père dans la culture vietnamienne des exilés}

La figure du père vietnamien doit au préalable être rapportée à la notion de " chef de famille ", qui se comprend elle-même à partir de la pratique du culte des ancêtres. On peut dire qu'il y a deux sortes de famille, et par conséquent deux sortes de chefs de famille, celles des aînés et celles des cadets. Dans les secondes, il n'y a pas, en principe, de culte des ancêtres. Le cadet est destiné, sauf exception, à devenir un chef de famille au sens restreint. Toutefois, en pratique, chaque chef de famille pratique le culte des ancêtres et dispose dans sa maison d'un petit autel pour leur rendre hommage. Ce culte domestique s'intègre symboliquement dans le cadre d'un culte des ancêtres à l'échelle de la famille étendue, où l'aîné d'une branche exerce son autorité sur tous les membres de cette branche. A la mort du père, l'aîné devient le "Maître du deuil ". Il préside les cérémonies funéraires et opère les rites (Cadière, 1992, p. 54). Autrefois, pour assumer ses responsabilités de chef du culte, le Trưởng tộc, "le premier, le chef du clan familial », disposait de ressources qui lui permettait d'entretenir les tombeaux et de réaliser les offrandes, les biens Hương hỏa, la " part de l'encens », littéralement, " pour l'encens et le luminaire ». C'était une des institutions les plus sacrées. Ce chef de famille exerçait une grande influence morale : il arbitrait les grandes réunions de famille et les conflits d'intérêt, réglait les affaires administratives (testaments, contrats d'aliénation de terrains), approuvait les mariages, etc. (p. 57 et p. 61).

Ce qu'il faut retenir de ces données - qui, en reflétant davantage un ordre normatif que la réalité pratique, n'échappent pas à un certain schématisme culturaliste - pour appréhender la situation contemporaine, c'est que le père est le garant symbolique parmi les membres de la maisonnée des valeurs filiales, l'intermédiaire entre les ancêtres et les vivants, et qu'à ce titre, il incarne la figure de l'autorité (Wabled, 2001, p. 97).

En 2005, à la pagode de Bagneux, j'ai rencontré et observé Diên, un jeune arrivé en France à l'âge de dix-sept ans au début des années 2000. II venait rendre un hommage anniversaire à son défunt père, accompagné de son oncle et de sa tante. Il présente un profil intéressant, car ses pratiques religieuses et culturelles façonnées en dehors du contexte migratoire relèvent de modalités de subjectivation différentes de celles des enfants d'exilés de son âge :

"Quand mon père est mort (au Vietnam), j'ai dû assumer mon statut de fils héritier. Je devais porter le deuil en revêtant une combinaison blanche, un ruban et un chapeau blancs ${ }^{1}$, et une canne en bambou. Les gens se prosternaient devant le défunt, et moi, je devais recevoir leurs condoléances et me prosterner à mon tour; je recevais donc les hommages et je les remerciais. Ma mère $m$ 'avait expliqué le protocole et parfois un oncle me donnait des indications en chuchotant. [...] Les enfants de ma tante numéro sept en France, un garçon et une fille; eux, à la pagode, ils participent au rituel par obligation. Ils ne sont pas trop influencés par la culture vietnamienne et parlent peu la langue. » (Diên, 21 ans, étudiant, célibataire)

La filiation père/fils aîné est consacrée à travers l'institution des rites de mort et la transmission du statut de chef de famille ne relève pas tant d'un engagement conscient, délibéré, si l'on peut dire, que d'un engagement infraconscient privilégiant le rapport au corps. Le père reste présent en tant que défunt. Diên a intériorisé un sentiment de dette. Il agit sur le mode du devoir être, du rôle à jouer, de l'obéissance à la règle. C'est un type de transmission très spécifique dont il faut brièvement rendre compte si l'on veut traiter la question de l'engagement paternel dans ce contexte post-migratoire. À mon sens, il est utile de se référer à la notion bourdieusienne de mimétisme ${ }^{2}$, telle

\footnotetext{
${ }^{1}$ Voir Gidoin (2014a) pour la signification de la couleur blanche.

${ }^{2}$ La notion de mimesis en tant que processus d'acquisition, renvoie elle-même aux notions fondamentales d'hexis corporelle et d'habitus, l'idée étant que les individus intériorisent des postures, des dispositions du corps par un processus d'incorporation qui implique un rapport global d'identification et qui s'accomplit en deçà de la distance réflexive. Ces dispositions fonctionnent comme des principes inconscients d'action et de perception (Bourdieu, 1980, p. 123)
} 
qu'elle a été reprise et mise en perspective dans le contexte de la culture vietnamienne par Pottier, c'est-à-dire à un certain mode de subjectivation par le corps à travers les rites :

"L'intériorisation des valeurs morales sur lesquelles repose l'harmonie de la vie familiale passe par l'incorporation de comportements strictement codifiés qui recouvrent à la fois des manières de s'exprimer et des attitudes corporelles. " (Pottier, 1999, p. 47)

"L'essentiel pour le sujet confucéen, qui a intériorisé les normes et les principes régulant les relations sociales, est de savoir se conformer aux modèles du comportement correct. » (Pottier, 2007, p. 47-48)

Diên, au Vietnam, de même qu'en France à la pagode, obéit scrupuleusement au formalisme rituel. II se soucie de bien faire comme s'il accomplissait un devoir. Ses propos éclairent, par contraste, certaines spécificités des hommages aux défunts revisités à partir du bouddhisme vietnamien en France, lesquelles, comme nous allons le voir par la suite, participent à l'élaboration de nouvelles modalités de transmission paternelle. L'observance des rites chez ce jeune exilé se démarque de l'attitude de la plupart des jeunes que l'on peut observer dans les pagodes de la région parisienne. Chez lui, au Vietnam, le modèle du culte des ancêtres familial opérait encore suffisamment et ne nécessitait pas d'alternative rituelle compensatoire comme c'est le cas en France. La maison demeurait, au Vietnam, et semble rester, en France, chez son oncle, le "théâtre " principal de la vie religieuse. À la mort de son père, il se sent naturellement investi du rôle social qui lui incombe : être le fils aîné. Une tendance qui, comme j'ai pu le constater sur le terrain, décline dans le contexte français, notamment chez les jeunes dont les parents sont d'origine vietnamienne. Car le formalisme confucéen s'est érodé avec le temps et se trouve dans une situation de crise (Gidoin, 2014b, p. 50-51).

\section{Transmettre en déléguant la charge du culte des ancêtres à la pagode}

À partir de témoignages de jeunes fréquentant la pagode, intéressons-nous au rôle joué par le père de famille dans cette délégation et réinterprétation du culte des ancêtres.

" Je suis venu vivre en région parisienne pour le travail. J'accompagne aujourd'hui mon oncle et ma tante chez qui j'habite. Avec mes parents, j'allais à la pagode de Toulouse au moins une fois dans l'année, au Nouvel An pour rendre hommage aux défunts. Je pense qu'en suivant mes parents à la pagode, ils m'ont transmis quelque chose des principes moraux vietnamiens. Honnêtement le bouddhisme, la réincarnation, tous ces trucs, moi j’y crois. " (Samuel, 21 ans, vendeur)

Au-delà de l'aspect spécifiquement lié à la relation aux défunts, le fait de transférer le culte des ancêtres à la pagode, et d'être ainsi affilié au bouddhisme vietnamien en France, offre un dispositif par l'intermédiaire duquel peuvent légitimement être transmise certaines valeurs morales fondamentales aux yeux des Vietnamiens exilés, en particulier celle de la "piété filiale »(Gidoin, 2013, p. 198-199). La fréquentation occasionnelle de la pagode, souvent devenue coutumière en France, parce que les jeunes accompagnent depuis leur enfance leurs parents pour les hommages aux ancêtres, crée le sentiment d'une appartenance communautaire, de même qu'un lien d'attache affectif au bouddhisme. Notons que l'aménagement de cet espace d'entre-soi social et culturel «à la vietnamienne » résulte de l'initiative des deux parents.

" Je vais à la pagode avec ma famille tous les trois mois quand il y a un hommage anniversaire à un défunt, comme aujourd'hui pour mon père (décédé récemment). À la maison, on continue de pratiquer le culte des ancêtres. Ces deux cultes restent présents pour moi. J'ai le sentiment que mes deux parents ont joué le même rôle au niveau de la transmission culturelle et religieuse. En France, sans la pagode, je crois que les gens seraient de moins en moins dans leur culture. [...] Je me sens moins impliqué que mes parents dans le bouddhisme vietnamien; eux sont plus dedans, alors que moi j'ai plus de recul avec la culture occidentale. Pour l'instant je me sens plus impliqué dans le culte des ancêtres, mais j'accompagne mes parents à la pagode quand ils me le demandent. En même temps, je crois que je me sens bouddhiste. [...] Tout se fait naturellement. Dès qu'on est petit, nos parents nous amènent à la pagode. » (Alexandre, 16 ans, lycéen)

On perçoit dans les propos d'Alexandre le rôle décisif de la pagode en France comme lieu de transmission de la culture et de la religiosité vietnamiennes; mais il n'en a pas forcément conscience. II est probable que le culte des ancêtres domestique, par rapport auquel il déclare se sentir davantage impliqué, se perpétuerait difficilement s'il n'était pas rattaché à un culte périodique d'hommage aux défunts à la pagode. On constate cependant une prise de 
distance par rapport à la dimension ethnique en raison de l'influence de la culture occidentale. La transmission dans un contexte d'acculturation implique des mutations du sentiment religieux. II ne faut toutefois pas négliger la part d'éducation à la vietnamienne que reçoivent ces jeunes, et qui semble être favorisée par l'affiliation à la pagode : inculcation de la culture et de la religiosité dès la prime enfance. Le fait d'accompagner ses parents à la pagode est une constante que l'on retrouve dans quasiment tous les témoignages des jeunes; en terre d'exil, cela participe d'une stratégie éducative. La transmission se "fait naturellement ", de façon inconsciente, dans la continuité du modèle traditionnel axé sur le mimétisme.

À la pagode, le père n'occupe plus le devant de la scène. Ce que l'on peut en déduire, c'est un amenuisement du sentiment d'appartenance au " clan familial » et un affaiblissement de l'autorité paternelle traditionnelle. II faut voir que la famille vietnamienne, dans le contexte post-migratoire, connaît un processus d'acculturation aux valeurs de la famille française (et plus globalement occidentale) contemporaine. Cette dernière se caractérise, pour simplifier, par le fait que les individus ont davantage leur logique propre (divergences individuelles), ce qui a pour effet de limiter le « communisme familial » (de Singly, 2005, p. 11). Les solidarités, jadis ritualisées, s'assouplissent dans le cercle de parenté. On tend à passer du régime de la contrainte à celui de l'élection; l'affinité fonde la solidarité. Les représentations traditionnelles de la division sexuelle du travail entre les conjoints deviennent illégitimes; sans disparaître, les rôles se redéfinissent. L'idéologie moderne implique les notions d'égalité, de partage, notamment dans le "domaine des décisions ». II en résulte que la suprématie du père est battue en brèche "parce qu'elle contrarie les exigences modernes" (p.103) : le père n'est plus le chef incontesté de la famille; on sort par conséquent du système patriarcal.

«Ce sont mes parents au début qui ont voulu. Quand j'étais enfant, mes parents m'emmenaient déjà à la pagode, occasionnellement. [...] Pour être honnête, tout ce qui est religieux, je n'y connais rien. [...] La pagode se développe, avant, il n'y avait pas autant de monde (il fait allusion à la pagode d'Évry ${ }^{3}$ ); maintenant on reçoit le dalaï-lama (en 2006 et 2008). [...] La piété filiale, ça ne me parle pas trop, même si je rends régulièrement hommage devant les autels à la maison (un pour les ancêtres et un autre pour le Bouddha), par tradition ou plutôt par respect envers mes parents (offrandes d'encens et prosternations); je le fais deux trois fois par mois. » (Albert, 24 ans, ingénieur, célibataire).

Albert donne le sentiment de ne pas être impliqué, obéissant avant tout au désir de ses parents. On constate cependant que la fréquentation de la pagode, quand elle participe d'une stratégie éducative, même souple, crée un sentiment d'appartenance, qui renvoie à ce que l'on pourrait appeler un bouddhisme " affectivo-patrimonial ". II n'est d'ailleurs pas peu fier de faire allusion à la réception du dalaï-lama à la pagode d'Evry, signe tangible de l'expansion du bouddhisme vietnamien. Notons qu'en disant "on ", il s'identifie à sa façon à un "nous les bouddhistes vietnamiens $"$.

\section{Jouer son rôle de chef de famille vietnamien dans un contexte d'acculturation}

À la pagode de Joinville-le-Pont, j'ai suivi, durant sept semaines (première période de deuil), une famille vietnamienne qui venait pour des cérémonies de deuil hebdomadaires. En principe, la mort s'accompagne de quatre moments importants pour le défunt et sa famille: la cérémonie funéraire au moment du décès, les cérémonies du $49^{\mathrm{e}}$ et du $100^{\mathrm{e}}$ jour et l'anniversaire de la date de la mort.

Par l'intermédiaire d'un jeune, le fils aîné du fils aîné du défunt, j'eus un accès privilégié à mon terrain d'enquête. Cette situation m'obligea cependant à faire preuve de réflexivité. Porter un regard réflexif sur une enquête ethnologique en train de se faire, cela signifie d'abord, comme le rappelle F. Laplantine, que

nous n'observons jamais les comportements d'un groupe tels qu'ils auraient lieu si nous n'étions pas là ou si les sujets de l'observation étaient d'autres que nous. De plus si l'ethnographe perturbe une situation donnée, et même crée une situation nouvelle, due à sa présence, il est à son tour éminemment perturbé par cette situation. Ce que vit le chercheur dans sa relation à ses interlocuteurs (ce qu'il refoule, ou ce qu'il sublime, ce qu'il déteste ou ce qu'il chérit), fait partie intégrante de sa recherche. (Laplantine, 2005, p. 25)

\footnotetext{
${ }^{3}$ Au milieu des années 1990, l'association bouddhique de Bagneux a entrepris de construire une grande pagode à Évry, afin de disposer d'un haut lieu d'enseignement, de formation des moines et d'échange entre bouddhistes. C'est aujourd'hui la plus grande pagode d'Europe, toutes obédiences confondues.
} 
Les relations entre les individus dans la culture vietnamienne sont encore souvent soumises au respect de la hiérarchie. Du fait de mon âge, j'ai été assimilé dans ce contexte familial à la génération des enfants. S’il s'en fallait de peu pour que certaines personnes acceptent spontanément de répondre à mes questions, toutes se sont finalement habilement arrangées pour trouver une échappatoire en prétextant le manque de temps. Paradoxalement, leurs refus me sont apparus comme légitimes. Laurent assumait donc à mon égard - l'autrui étranger au cercle du lignage, et qui plus est, observateur prenant des notes - le rôle du médiateur-informateur que lui avait implicitement attribué ses proches. Laurent leur expliqua les raisons de ma présence, ce qui me permit d'être intégré dans l'espace de l'intimité familiale et $m$ 'apporta des éléments de compréhension sur les arcanes du culte. Ce jeune m'a déclaré lors de notre rencontre qu'il venait à la pagode uniquement pour rendre service à ses parents qui n'avaient pas le permis de conduire. Or mes observations dans la longue durée, axées en particulier sur les conduites motrices, allaient au contraire montrer que ces cérémonies présentaient pour lui un grand enjeu symbolique, que l'on peut formuler de la manière suivante: que faire de l'héritage symbolique de la filiation? Et cette problématique devait s'avérer fondamentale pour la suite de mon enquête. Je pris alors conscience des limites de la dimension verbale à pouvoir signifier un savoir pratique, qui agit de manière infra-consciente, ce " sens pratique » théorisé par Bourdieu (1980), obéissant à diverses stratégies sociales, et difficile à saisir de prime abord.

« Mon père, c'est la première fois qu'il allait à la pagode. II n'est pas très pratiquant. Ma mère, elle, a de vraies convictions religieuses. Mon père a un peu accepté pour lui faire plaisir. Ma mère a un petit autel consacré au Bouddha dans le salon, mais pour moi et mon père, il fait partie du décor; nous, on ne pratique pas. Dans la famille, ce sont les femmes qui pratiquent. [...] Être l'aîné, c'est important. Ses frères et sœurs, ma mère aussi, le poussent pour qu'il joue son rôle d'aîné. " (Laurent, 28 ans, étudiant)

Poussé sur la scène rituelle par son entourage, le père de Laurent est amené à jouer un rôle social et symbolique, celui du "chef du culte des ancêtres ». Mais les valeurs morales confucéennes, après plusieurs décennies de vie occidentale, n'ont plus la force de l'évidence. La ritualisation du deuil à la pagode, voulue par la mère de Laurent, vise, me semble-t-il, à instaurer un ordre à un moment clé de l'histoire familiale. En cette occasion où le groupe familial doit objectiver et redéfinir sa cohésion, le rôle du père de Laurent est décisif. Le surgissement de la mort du patriarche introduit dans le réel une temporalité de la tradition, qui ne va plus tellement de soi en temps ordinaire, mais qui impose à ce moment-là la question de la transmission intergénérationnelle, et plus particulièrement celle entre un père, fils aîné du défunt, et son propre fils aîné.

Au cours de mon enquête, je m’étais demandé ce qui pouvait inciter Laurent, un jeune de la «troisième génération » (le seul parmi les trente-deux petits-enfants à accompagner ses parents à la pagode chaque semaine) à participer à un rituel funéraire relevant de pratiques religieuses dont il ignorait en partie la signification. D'autant que la relation assez libre qu'il entretenait avec son père - dont l'attitude s'opposait délibérément à la figure traditionnelle du père vietnamien autoritaire et distant - ne semblait a priori l'y contraindre d'aucune manière. Je constatai tout d'abord qu'il se sentit, en tant que petit-fils aîné du défunt, en principe héritier légitime du lignage pour la troisième génération, investi du devoir de m'informer de certaines choses concernant le rite auquel il allait participer : « Le grand-père nous a nourris, maintenant, en retour, on lui fait des offrandes. »

je remarquai que les membres de la deuxième génération, c'est-à-dire les parents, oncles et tantes de Laurent, même s'ils manifestaient un manque de maîtrise du rituel bouddhique, témoignaient par leur gestuelle d'un conditionnement corporel intrinsèque relevant des pratiques religieuses vietnamiennes "ordinaires »" Les cérémonies à la pagode étaient ainsi l'occasion pour le petit-fils de s'initier à toutes ces pratiques de base. Au moment de s'engager dans le rituel, le mimétisme à l'égard de la deuxième génération s'affirmait nettement. Son attention était retenue par l'image du défunt et par les manipulations qu'effectuait son père. Dans cette atmosphère confinée, les liens familiaux se resserraient. Laurent observait son père " jouer son rôle d'aîné ". En accompagnant chaque semaine ses parents à la pagode, en particulier son père, qui était sur le devant de la scène

\footnotetext{
${ }^{4}$ Savoir montrer sa déférence envers une personne hiérarchiquement supérieure (en l'occurrence ici les moines et les nonnes, mais valable aussi en dehors du contexte religieux, en famille par exemple) en inclinant son buste, effectuer des offrandes en brûlant des baguettes d'encens et faire des lạy pour rendre hommage à telle ou telle divinité (au Bouddha et aux différents bodhisattvas).
} 
rituelle, il était amené à pratiquer « une gymnastique symbolique dans laquelle le corps pense pour lui » (Bourdieu, 1980, p. 123).

« La première fois que je suis allé à la pagode, j’ai été assez surpris par les rites. Pendant un mois, j'ai ressenti un poids, j'étais nerveux et j'avais du mal à dormir. Au fur et à mesure des cérémonies, ça s'est apaisé. Je ne m'attendais peut-être pas aux conséquences de cet événement. Aujourd'hui, je ne me pose pas la question de savoir ce que j'aurais dû faire. "

Dès lors que Laurent décide de s'engager dans le rituel à travers les conduites motrices qu'il exige, les contenus verbaux deviennent très secondaires et ce sont les processus inconscients de symbolisation qui deviennent prédominants. Il active les schèmes de la religiosité des relations intrafamiliales qui lui ont été transmis dans son enfance. Ces schèmes ont pu rester dans un état de latence, car les jeunes de la troisième génération sont le plus souvent façonnés par de multiples modalités de subjectivation. Raison pour laquelle il faut éviter ici d'adopter une conception linéaire du processus d'acquisition (Parlebas, 1981, p. 81) et de transmission. Le rapport de Laurent à la langue d'origine de ses parents est à ce propos révélateur. Il me confiait que dans les premières années de sa vie il y était sans cesse exposé, même si en parallèle il arrivait aux parents de parler français. Puis, avec la scolarisation, la langue française devint petit à petit dominante.

Ce jeune participe à des cérémonies de deuil qui redéfinissent sa place au sein de la famille, le propre du rite étant d'instituer un ordre.

" J'ai une grande sœur, mais c'est moi que l'on met en avant ${ }^{5}$. Ces cérémonies ont fait émerger ce sentiment d'avoir un rôle à jouer. Je me suis senti obligé d'assister aux cérémonies, pour moi, mes parents, mon père et mon grand-père. [...] Maintenant, je parle beaucoup plus avec l'oncle numéro six, le plus porté sur la religion, et celui qui ressemble le plus à mon père; avant c'était la relation adulte-enfant. Je ne sais pas si ça va avoir de l'importance le fait que j'accède à un nouveau niveau dans la parenté avec la mort du grand-père. En privé, mon père m’a dit merci. Je lui ai posé des questions pour en savoir plus sur nos origines et ne pas rester dans l'ignorance. Ce sont des choses à voir une fois dans sa vie parce que mes parents voudront peut-être que je le fasse pour eux un jour. Je le fais pour faire quelque chose avec mes parents. »

Son père, en étant amené à jouer son rôle de fils aîné (un peu malgré lui), transmet à son fils, de fait, le sentiment d'avoir lui-même un rôle à jouer, et l'idée que la construction de soi passe par une assomption de sa place au sein du groupe familial. Avec la mort du patriarche, et en accompagnant son père à la pagode, il accède symboliquement au monde des adultes. Plus fondamentalement, il participe au renouvellement générationnel, qui implique une prise de connaissance des "origines pour ne pas rester dans l'ignorance ", de même qu'une projection dans l'avenir, quand les parents avanceront en âge. L'engagement paternel consiste à montrer l'exemple. Laurent imitera son père et montrera l'exemple à son niveau, vis-à-vis de ses cousins et cousines. Cependant, il manifeste aussi une distance, une prise d'autonomie, à travers des propos assez occidentaux : « le faire au moins une fois dans sa vie ". II ne conçoit pas la filiation comme une allégeance à la lignée; il éprouve même un sentiment d'étrangeté par rapport à la hiérarchisation des relations intrafamiliales, mal vécue parce qu'elle fait de moins en moins sens aujourd'hui :

"Souvent, lors des réunions de famille, je me sens écrasé par le nombre. Quand tu es un jeune, on ne tient pas compte de ce que tu peux raconter. II y a une coupure entre les générations. J'ai tendance à rejeter ce système. »

Laurent se sert tendanciellement de la mémoire familiale comme d'un capital symbolique, une ressource actualisable pour l'existence présente et future (Déchaux, 1997, p. 264 et 271). Cependant, notons qu'une forme de rupture avec le ritualisme traditionnel existe déjà au niveau de la deuxième génération. Le meilleur exemple, c'est bien sûr celui du père de Laurent. Pourtant, en privé, ce dernier ne manque pas de remercier son fils d'être venu à toutes les cérémonies, contrairement à ses cousins. L'ambivalence de son attitude vient probablement de la négociation - déjà présente pour cette génération - entre une liberté individuelle et un respect de la tradition. L'ordre de la famille vietnamienne traditionnelle a perdu de son sens et de son efficience. Les rapports entre hommes et femmes, maris et épouses, ont évolué (Vu-Renaud, 2002, p. 37-38) : alors qu'au Vietnam le rôle des femmes dans le culte des ancêtres restait la plupart du temps secondaire, il peut devenir en France déterminant.

\footnotetext{
${ }^{5}$ Les filles, si l'on peut dire, « comptent pour du beurre » dans la tradition confucéenne.
} 
Ainsi, la mère de Laurent, bien plus qu'une assistante, a pris l'initiative d'organiser les hommages à la pagode, et guide son mari durant les cérémonies. Cette influence de la mère à un moment où le père est censé assumer son statut de "Maître du deuil » me paraît innovante. Et dans la mesure où elle est la seule véritable pratiquante bouddhiste de la famille, on peut surtout se demander si elle ne promeut pas dans le même temps son affiliation au bouddhisme et les valeurs qui s'y rattachent. La mère propose un nouveau cadre social, la pagode - à un moment où l'on observe que de plus en plus de Vietnamiens redécouvrent le bouddhisme en terre d'exil -, pour redéfinir et réinterpréter les valeurs fondamentales de la culture d'origine. II y aurait là un usage social de la relation rituelle confucéenne défunt-fils aîné, associée à la figure paternelle, au bénéfice du référent bouddhique, ici associé à la figure maternelle. Et les moines, en prenant en charge le culte ${ }^{6}$, en se substituant au chef de famille, dépossèdent en quelque sorte, avec son consentement implicite, le fils aîné de son statut symbolique de chef du culte des ancêtres.

\section{La « famille bouddhique " comme nouvelle lignée imaginaire de la transmission}

Je m'appuie ici sur les témoignages de M. Phuoc, un retraité bénévole de la pagode de Bagneux, et de son épouse. $M$. Phuoc m'a toujours témoigné beaucoup de bienveillance, et me présentait souvent aux fidèles. "Ici, c'est une grande famille! ", aimait-il à dire. M. Phuoc répétait souvent d'un air placide qu'il connaissait pratiquement tout le monde, que ce soit les habitués ou les familles qui fréquentent la pagode selon le cycle des dates anniversaires de la mort des défunts. L'entremise de M. Phuoc favorisa mon intégration au sein de la pagode. Il se peut aussi qu'il projetât sur moi l'image du fils (ou du petit-fils) idéal, adoptant par conséquent la posture du père vietnamien transmetteur de valeurs. M. Phuoc savait que j'avais des origines vietnamiennes par mon père, et de surcroît que je m'intéressais à ce qui lui tenait désormais le plus à cœur, le bouddhisme. Quoi qu'il en soit, il cherchait à me faire partager sa vérité, la voie du Bouddha, qu'il avait redécouverte en France au moment de la retraite.

L'objet religieux est spécifique, la question de l'intégration pose problème parce qu'il y a souvent une tension entre l'engagement dans la religion par la foi, qui caractérise la posture des gens que l'on observe, et la posture désengagée, critique, qui caractérise la posture réflexive du chercheur. Les bonnes et mauvaises surprises que lui réservent les relations avec les acteurs, informateurs privilégiés ou non, sont généralement dues à un rapport transférentiel. De nombreuses personnes, souvent à la retraite, se sont ainsi comportées à mon égard comme des ambassadeurs de cette culture vietnamienne réinterprétée à partir du bouddhisme. Mon statut de chercheur y était pour beaucoup : il influençait la façon dont elles se comportaient et, par conséquent, la réalité qu'il m'était donné d'observer et de vivre ${ }^{7}$. Le principal enseignement que j'en ai tiré, c'est que les gens de cette génération ressentent un désir de transmission intergénérationnelle, qui peut s'expliquer par un déficit en la matière au sein de leur groupe familial, le culte des ancêtres domestique étant de moins en moins pratiqué. Cette carence est compensée par la redécouverte du bouddhisme et la réinterprétation du culte des ancêtres en termes bouddhiques.

«Maintenant, en France, comme je suis à la retraite, j'ai du temps. Je trouve que notre bouddhisme aujourd'hui est différent de ce que l'on faisait avant avec nos parents. Ici, avec les enseignements du vénérable de la pagode de Bagneux, $c^{\prime}$ est plus profond ${ }^{8}$, moins intéressé; le bouddhisme, $c^{\prime}$ est une philosophie. Quand on est jeune, il faut construire une famille, avoir un boulot, on a des soucis et on ne pense pas à autre chose. Depuis, ma vie a complètement changé, je suis devenu calme, non violent. [...] J'ai trois enfants, un garçon et deux filles. L'aîné, le garçon, est né en 1960 et la benjamine en 1963. La cadette est sage-femme et la dernière urologue. Eux, ce n'est pas la même mentalité, à chaque fois que je leur en parle, ils disent que c'est du "blabla »; ils sont cartésiens. Ma femme est bouddhiste également, mais pas trop pratiquante. Nous les bouddhistes pratiquants, contrairement aux matérialistes, nous préparons notre bonheur futur. [...] Au Vietnam, dans la coutume, le culte des ancêtres se transmet de père en fils. À la maison on allumait juste les baguettes d'encens pour les ancêtres. Mon père était bouddhiste mais non pratiquant. [...] Maintenant, en France, le culte des ancêtres, c'est autre chose. Le culte des ancêtres traditionnel, c'est erroné! [...] Ici, on a l'occasion d'approfondir les enseignements bouddhiques. Mes parents sont morts, ils sont à la pagode, avec mon frère aussi. De leur vivant, nous

\footnotetext{
${ }^{6}$ En effectuant quotidiennement des prières durant les 49 jours de deuil, avec l'éventualité après, pour la famille, d'une délégation définitive du culte, ce qui ne sera pas le cas pour la famille qui viendra récupérer les cendres du défunt.

${ }^{7}$ Ces effets de situation ont été très bien analysés par Richard Pottier (2007, p. 104-108).

${ }^{8}$ On retrouve très souvent la même remarque chez les bénévoles retraités : c'est en effet en France, souvent, qu'ils seraient devenus véritablement bouddhistes.
} 
n'avions pas trop d'occasions pour montrer notre reconnaissance. Alors aujourd'hui, quotidiennement, on leur rend hommage. Quand il y a un membre de la famille qui est bouddhiste, cela favorise le salut de la famille. " (M. Phuoc, 75 ans, retraité, bénévole à l'association de la pagode de Bagneux)

"Moi j'ai été initiée à la méditation bouddhique. Nous nous sommes un peu disputés à l'époque. Pour qu'il puisse suivre sa voie librement, j'ai décidé de laisser la pratique de la méditation de côté. Pour moi, c'est la paix de la famille le plus important. La morale me dit de ne pas dire de mal de mon mari. [...] Mes enfants, comme ils ont été élevés en France, ils ont tendance à dire que le bouddhisme de leur père à la pagode de Bagneux, c'est une secte. Mon fils Jean-Philippe, qui a 50 ans, a épousé une Vietnamienne en France. Elle est bouddhiste. C'est d'ailleurs la famille de mon mari qui [la] lui a présentée. J'ai deux filles, une a épousé un Corse et l'autre un Algérien. [...] Je crois que mon fils a hérité de son père, même s'il ne se sent pas trop impliqué par le bouddhisme vietnamien pour le moment. Avant, quand il était jeune, il parlait le vietnamien, mais après il a laissé tomber. Avec les petits-enfants, on a pris l'habitude de parler français avec eux, donc ils ne parlent pas le vietnamien; on a sûrement eu tort. » (Mme Loan Anh épouse de M. Phuoc, retraitée)

M. Phuoc n'aime pas parler de lui, par pudeur. C'est une attitude caractéristique des pères vietnamiens de cette génération qu'il faut souligner pour appréhender la question de l'engagement paternel dans transmission de la culture vietnamienne en terre d'exil. Grâce au témoignage de son épouse, on voit que son fils s'inscrit dans la filiation patrilinéaire "à la vietnamienne ", ce que ne laissent pas supposer les propos de son père. Autant ses filles ont opté librement pour la mixité culturelle, autant son fils, en se mariant avec une femme vietnamienne, bouddhiste de surcroît, perpétue l'attachement à une certaine tradition. Le dénigrement de la jeunesse de la part de M. Phuoc fait partie d’une rhétorique classique attachée à la posture d'autorité du père vietnamien. Son épouse, malgré sa libre pratique du bouddhisme et les tensions que cela engendre vis-à-vis de $\mathrm{M}$. Phuoc, se conforme à la morale confucéenne: son mari est le chef de la famille et elle doit en priorité veiller à l'harmonie familiale. Attachée à son rôle de mère, elle ne semble pas mécontente que son fils ait hérité de son père.

Au Vietnam, l'enjeu fondamental de la transmission culturelle était indissociable de la pratique du culte des ancêtres qui véhiculait les valeurs de la piété filiale. En redécouvrant le bouddhisme en France, un bouddhisme différent de celui de ses parents au Vietnam, moins traditionnel, moins "intéressé ", au sens où l'on n'en attend pas délibérément des bénéfices mondains, $M$. Phuoc délaisse le culte des ancêtres traditionnel. Avec la vie en exil et l'influence du mode de vie occidental, ses rapports aux deux principaux référents religieux vietnamiens ne sont plus ce qu'ils étaient. Le bouddhisme, devenu plus spirituel, condense désormais pour ce retraité tous les enjeux de la transmission intergénérationnelle : rapport aux défunts, à la mort, à la religiosité et plus globalement à la culture vietnamienne. M. Phuoc a redéfini les enjeux de la transmission à partir d'une lignée imaginaire bouddhique, dont la figure emblématique est celle du grand vénérable de la pagode de Bagneux. Ce dernier a pour les fidèles l'aura d'un homme de grande sagesse qui connaît la culture et l'histoire des exilés, ainsi que leurs préoccupations actuelles, diverses et variées en fonction des générations et de la situation sociale de chacun. Par un effet de déplacement symbolique, le vénérable incarne l'autorité traditionnelle du chef de famille. Et si l'on se réfère aux représentations de $\mathrm{M}$. Phuoc, on peut concevoir l'imaginaire d'un esprit de famille, une nouvelle chaîne signifiante où, respectivement, le Bouddha, le vénérable, les bénévoles, et les jeunes fréquentant l'association, symbolisent les différents niveaux de la filiation : l'ancêtre, le grand-père, les parents et les enfants. Au niveau de la pratique, le moine (le vénérable n'exécutant pas les rites, les moines bénéficient de son aura symbolique au niveau de la pratique), en devenant l'opérateur des rites du culte des ancêtres, prend en quelque sorte la place du chef de famille.

II y a un commun accord entre les religieux et les fidèles sur l'idée qu'une part fondamentale de l' « héritage culturel ancestral » (pour reprendre les termes mêmes du vénérable) est axée sur les valeurs de la piété filiale. C'est une thématique qui, d'un côté, par son caractère universel (le lien de filiation) dépasse les contingences d'un contexte culturel particulier, et de l'autre, permet de rester dans une continuité et de revisiter la tradition. On passe alors de l'esprit de famille traditionnel à un esprit de famille bouddhique. Dans cette perspective imaginaire réaménagée, M. Phuoc, bien qu'il déclare rejeter la religiosité traditionnelle, montre qu'il reste en fait très attaché aux valeurs de la piété filiale.

Pris au pied de la lettre, ce réaménagement imaginaire peut aboutir à une sortie de la lignée bouddhique vietnamienne, et par conséquent à un désinvestissement de la dimension ethnico-religieuse : 
"Mon fils s'est engagé dans le bouddhisme tibétain ${ }^{9}$ après l'obtention de son diplôme d'ingénieur. II ne se sentait pas trop concerné par la dimension typiquement vietnamienne à la pagode de Bagneux; je pense que c'est parce qu'il a grandi en France. " (M. Thuông, 55 ans, ingénieur, bénévole à l'association bouddhique de Bagneux)

\title{
Affiliation et transmission bricolées : quand les enfants et petits-enfants d'exilés deviennent pères
}

\begin{abstract}
« Mon mari et moi sommes plus ou moins athées. Le père de mon mari est bouddhiste (sa mère est catholique) mais lui ne se sent pas particulièrement bouddhiste. Notre premier enfant est mort trois jours après sa naissance. On voulait une cérémonie, quelque chose parce que c'était très dur pour nous. On ne pouvait pas quitter notre enfant comme ça. Cela peut paraître surprenant, mais nous avons décidé d’organiser une cérémonie funéraire à la pagode de Bagneux. Notre fils a donc été baptisé; il est bouddhiste. Je pense que cela nous a aidés à surmonter cet événement, le plus douloureux de notre vie. Je pense que mon mari a joué un rôle sur moi. Comme je n'ai pas de religion, je me suis dit pourquoi pas. Depuis 2009, on vient deux fois par an à la pagode, une fois pour l'hommage anniversaire de notre fils et une autre fois pour le Têt, le Nouvel An vietnamien. Sinon à la maison nous pratiquons un culte des ancêtres à notre sauce. On a un autel dans notre salon sur une planche Ikéa : il y a un bouddha au milieu et des photos autour, nos grands-parents. Pour moi aussi ça fait sens, je m’y retrouve. En revanche nous ne faisons pas de prière comme les Vietnamiens. Nous avons deux filles de trois et huit mois; elles feront ce qu'elles veulent. Elles sont petites, mais la grande connaît déjà les gestes : incliner le buste et brûler une baguette d'encens. » (Emmanuelle, 30 ans, mariée, institutrice)
\end{abstract}

Chez ce couple mixte, le mari joue un rôle important. Avec sa culture vietnamienne quelque peu bricolée, il influence sa femme et bien sûr ses filles. II vietnamise en quelque sorte sa famille. II réalise pour cela des compromis qui aboutissent à une culture et à une spiritualité métisses. La référence à son père vietnamien est manifeste dans les propos de son épouse. D'après cette dernière, il ne se sentirait pas particulièrement bouddhiste, contrairement à son père - ce qui n'a rien d'outrageant, parce que le plus important dans la transmission du père au fils chez les Vietnamiens, c'est le culte des ancêtres. Pourtant, il va mobiliser le référent bouddhique pour affronter l'événement le plus douloureux de sa vie.

Transmission des gestes du culte des ancêtres

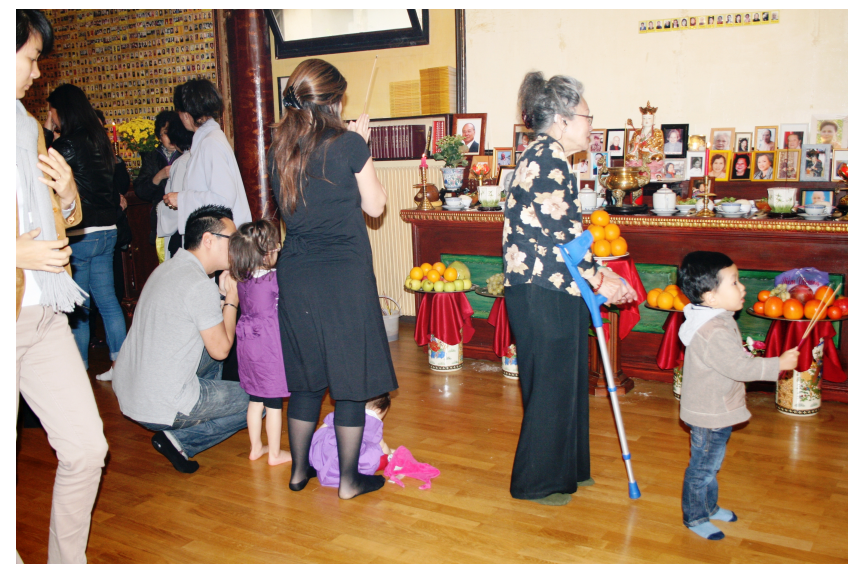

C'est qui est intéressant, c'est l'intégration d'une dimension universelle dans le bouddhisme et, le plus étonnant, dans la pratique du culte des ancêtres, si bien que ce dernier fait sens pour son épouse qui le pratique elle aussi, à sa manière. On retrouve dans cette culture métisse, d'une part, le mode de transmission aux enfants par mimétisme, à partir de gestes rituels de base : incliner le buste et brûler des baguettes d'encens, et d'autre part, la pagode comme lieu où s'exprime et s'élabore de façon inconsciente un rapport à la culture vietnamienne.

\footnotetext{
${ }^{9}$ C'est ce bouddhisme tibétain, avec un noyau Vajrayâna pour les adeptes les plus avancés, qui attire le plus grand nombre de sympathisants français, notamment la branche (il y en a quatre) Guelup-pa, l'école du dalaï-lama, qui bénéficie d’un prestige grandissant depuis que le prix Nobel de la paix lui a été décerné et qu'il vient régulièrement en Europe donner des conférences (en proposant un bouddhisme adapté à la vie occidentale). Voir Étienne et Liogier, 1997 (p. 57).
} 
En 2008, le jour du Vu Lan (la fête bouddhique de la piété filiale qui a lieu à la mi-août), j'eus l'occasion d'interroger Philippe, un jeune père de famille qui berçait sa petite fille de six mois :

« C'est la première fois que je viens à la pagode. Ma mère vient régulièrement le dimanche depuis qu'elle est à la retraite. [...] Moi, je viens d'emménager, il n'y a pas encore d'autel pour les ancêtres, mais je compte en installer un dans pas longtemps; pour perpétuer la tradition. C'est une marque de respect, une reconnaissance pour tout ce qu'ils ont fait; c'est aussi un lien à la culture d'origine. Depuis tout petit, on apprend à respecter les ancêtres; jusqu'à présent, ça n'a pas porté à préjudice donc... [...] Aujourd'hui, j'ai le temps; avant, j'étais beaucoup pris: le travail, le sport, je suis aussi pompier volontaire. [...] Je suis venu prier pour ma famille et pour ma petite fille. - Pour avoir la bénédiction des ancêtres? Oui, c'est un peu ça. » (Philippe, 28 ans, marié, technicien)

Il est étonnant et intéressant de constater que Philippe vient à la pagode pour la première fois à l'âge de 28 ans. L'investissement affectif préalable s'est fait par l'intermédiaire de sa mère (il ne me parla pas de son père). Le bouddhisme ne relève pas d'une tradition intériorisée dès l'enfance. C'est une expérience nouvelle pour lui. En revanche, il a " appris depuis tout petit à respecter les ancêtres ". Deux événements importants ont récemment marqué sa vie d'homme : il est devenu père et il a emménagé - deux rites de passage, si l'on peut dire, qui marquent le renouvellement générationnel. Investi de ce nouveau statut, il a ressenti le besoin de venir prier pour sa famille et sa petite fille; une façon, confirmait-il, d'avoir la bénédiction des ancêtres. Pourtant, soulignons-le, il n'a pas d'ancêtre à la pagode. À mon sens, l'essentiel est que cette dernière permet de raviver un imaginaire et une symbolique de la filiation, de renouer le fil générationnel en réinventant des modalités d'appartenance.

\section{Conclusion}

Dans le contexte post-migratoire français actuel, les familles vietnamiennes que j'ai observées en région parisiennes sont pour la plupart confrontées à une situation de mutation axiologique, consécutive au contact prolongé avec la modernité occidentale - caractérisée par l'individualisation et une tendance à la " déritualisation » de la vie sociale. On constate un rejet non de la philosophie confucéenne (qui est largement ignorée) mais du ritualisme confucéen. Cela conduit à une remise en question du patriarcat (aspiration à une nouvelle répartition des rôles sociaux entre époux, et contestation de la distinction aînés/cadets de la part des jeunes générations), et indirectement, une menace contre l'identité culturelle vietnamienne, dans la mesure où celle-ci est très liée au ritualisme, au patriarcat et aux valeurs confucéennes. Le bouddhisme permet dès lors, si j'ose dire, de "sauver les meubles ", au prix d'une réinterprétation des notions d'ancêtre et de pitié filiale, dont les gens n’ont en général pas conscience.

L'affiliation réelle et imaginaire à une communauté et à une lignée bouddhiques réinventées maintient un enracinement culturel auquel peut être combinée la revendication d'un ensemble de valeurs plus universelles, le bouddhisme étant de plus en plus considéré comme une doctrine philosophique en adéquation avec les valeurs de la modernité occidentale. Dans ce nouvel imaginaire de la transmission, le père n'occupe plus forcément le devant de la scène, quoique son influence reste perceptible et à vrai dire ambivalente, fluctuante selon les cas. On voit en tout cas émerger de nouveaux enjeux de transmission et corrélativement, de nouvelles façons d'être père et de transmettre en tant que père.

\section{Références bibliographiques}

Bourdieu, P. (1980). Le sens pratique. Paris : Minuit.

Cadière L. (1992). Croyances et pratiques religieuses des Vietnamiens ( 2 édition). Paris : École Française d’ExtrêmeOrient (3 tomes).

Condominas, G. (1953). Les sociétés indochinoises - Les Vietnamiens. Dans Leroi-Gourhan, A., Poirier, J. (dir.), Ethnologie de I'Union française (tome 2). Paris : Presses universitaires de France.

Déchaux, J.-H. (1997). Le souvenir des morts, essai sur le lien de filiation. Paris : Presses universitaires de France.

Durand, M. et Huard, P. (2010). Connaissance du Viêt-Nam. Paris : École Française d’Extrême-Orient, Réimpressions, $\mathrm{n}^{\circ} 12$. 
Étienne, B. et Liogier, R. (1997). Être bouddhiste en France aujourd'hui. Paris : Hachette.

Gidoin, J. (2013). Affiliation identitaire et affiliation eschatologique comme richesses primordiales chez les Vietnamiens en France. Dans C. Baroin, C. Michel (dir.), Richesses et société (p. 197-206). Colloques de la Maison René-Ginouvès, 8, Archéologie et Ethnologie.

Gidoin, J. (2014a). Le blanc, couleur du deuil dans la culture vietnamienne. Dans C. Coupry, F. Cousin (dir.), Les lumières du blanc, une facette de l'aventure textile (p. 147-155). Paris : Éditions Sépia.

Gidoin, J. (2014b). L'éthique familiale vietnamienne réactualisée par les jeunes générations à partir du bouddhisme en France. Enfances Familles Générations, 17, 46-57. (Transmission à rebours, filiation inversée, socialisation ascendante).

Kaufmann, J.C. (2004). L'entretien compréhensif. Paris : Armand Colin.

Laplantine, F. (2005). La description ethnographique. Paris : Armand Colin.

Olivier de Sardan, J.-P. (2013). La rigueur du qualitatif. Louvain-La-Neuve : Academia Bruylant.

Parlebas, P. (1981). Contribution à un lexique commenté en science de l'action motrice. Paris : INSEP, ministère de la Jeunesse, des Sports et des Loisirs.

Pottier, R. (2007). Yû Dî Mî Hèng "Être bien, avoir de la force ", essai sur les pratiques thérapeutiques lao. Paris : EFEO, monographie $n^{\circ} 192$.

Pottier, R. (1999). La place du corps dans les processus de socialisation : l'exemple du confucianisme. Dans M.P. Julien, J.P. Warnier (dir.), Approches de la culture matérielle. Corps à corps avec l'objet. Paris : L'Harmattan.

Singly, F. (de). (2005). Sociologie de la famille contemporaine. Paris : Armand Colin, $2^{\mathrm{e}}$ édition.

Vu-Renaud, M.-H. (2002). Réfugiés vietnamiens en France, Interaction et distinction de la culture confucéenne. Paris : L'Harmattan.

Wadbled, M. (2001). La transmission culturelle active dans les familles vietnamiennes en France. Hommes et Migrations, 1234, 95-102.

Alterstice - Revue Internationale de la Recherche Interculturelle, vol. 5, $n^{\circ} 1$ 